\title{
Gestión de las redes sociales y calidad de servicio electrónico en los hoteles de 3 estrellas del Cusco
}

\author{
Management of social networks and electronic service quality in the 3 star hotels of Cusco
}

\author{
Espinoza-Vilca, Sofía ${ }^{1}$; Espinoza-Sánchez, Raimundo ${ }^{2, \text { ”" }}$; Partida-Puente, Abel $^{3}$; Terán-Cázares, María Mayela ${ }^{4}$ \\ 1Universidad Autónoma de Nuevo León, Facultad de Contaduría Pública y Administración Monterrey, Nuevo León, México, \\ 2 Universidad Andina del Cusco, Facultad de Ciencias Económicas Administrativas y Contables Cusco, Cusco, Perú. \\ 3 Universidad Autónoma de Nuevo León, Facultad de Contaduría Pública y Administración, Monterrey, Nuevo León, México. \\ 4 Universidad Autónoma de Nuevo León, Facultad de Contaduría Pública y Administración, Monterrey, Nuevo León, México. \\ $\equiv$ raimundoespinoza@ hotmail.com
}

\begin{abstract}
Resumen
La presente investigación fue enfocada en las Pymes del sector hotelero de Cusco-Perú; hoy en día este sector productivo tiene mucha importancia para la economía del país, constituyendo un soporte significativo para el desarrollo empresarial y nacional. Las Pymes del sector hotelero se desarrollan en un entorno sumamente cambiante y competitivo, donde la tecnología digital tiene un papel protagónico modificando el comportamiento y la visión de los clientes, ya que estos cada vez son más informados y más exigentes con respecto a recibir un servicio electrónico de calidad. Es importante resaltar que hay una alta tasa de mortalidad en este sector productivo las cuales, en la mayoría de los casos, no llegan ni a los 5 años. En este contexto las organizaciones empresariales afrontan muchísimas dificultades, fundamentalmente en lo relacionado al mercado, ya que estas empresas tienen que afrontar una dura competencia y para hacerlo con éxito, una de las herramientas más poderosas es el uso de la mercadotecnia digital, enfocado en las redes sociales. El presente estudio tiene como objetivo sustancial analizar la incidencia de las redes sociales en la calidad del servicio electrónico en el sector hotelero de las Pymes de Cusco-Perú. Esta investigación es exploratoria, descriptiva, correlacional y explicativa, de enfoque cuantitativo. La recolección de datos se realizó en base a la investigación documental, bibliográfica y de campo, en el que se diseñaron y aplicaron 200 encuestas a los huéspedes que adquirieron los servicios de hospedaje vía electrónica. Los resultados mostraron que las redes sociales tienen una incidencia significativa en la calidad de servicio electrónico.

Palabras clave: Pymes, redes sociales, calidad de servicio electrónico.
\end{abstract}

\begin{abstract}
This research was focused on SMEs in the hotel sector of Cusco-Peru; these days, this productive sector is highly important for the country's economy, constituting significant support for business and national development. SMEs in the hotel sector is developed in a highly changing and competitive environment, where digital technology plays a leading role in modifying the behavior and vision of customers since they are becoming more informed and more demanding regarding receiving a service. Quality electronic. It is important to highlight that there is a high mortality rate in this productive sector which, in most cases, does not reach 5 years. In this context, business organizations face many difficulties, mainly concerning the market, since these companies have to face tough competition and to do so successfully, one of the most powerful tools is the use of digital marketing, focused on social networks. The purpose of this study is to analyze the impact of social networks on the quality of electronic service in the hotel sector of SMEs in Cusco-Peru. This research is an exploratory, descriptive, correlational and explanatory, quantitative approach. The data collection was carried out based on the documentary, bibliographic and field research, in which 200 surveys were designed and applied to guests who acquired the hosting services electronically. The results showed that social networks have a significant impact on the quality of electronic service.
\end{abstract}

Citar como: Espinoza-Vilca, S., Espinoza-Sánchez, R., Partida-Puente, A. y Terán-Cázares, MM. (2019). Gestión de las redes sociales y calidad de servicio electrónico en los hoteles de 3 estrellas del Cusco. Rev Yachay, 8(1),585-589.

Recibido: 20-11-2019; Aceptado 07-12-2019; Publicado: 12-12-2019

\section{Introducción}

Hoy en día, debido a la globalización vivimos en un mundo que está inmerso en la era digital y en las tecnologías de la información y comunicación; en este contexto las personas, clientes, organizaciones empresariales, instituciones, proveedores, compradores, competidores, entre otros, hacen uso de la tecnología digital cada vez con más frecuencia y de diversas maneras, las empresas utilizan cada vez más esta tecnología, tomando en cuenta la tendencia seguida por sus clientes que están cada día más conectados con el uso de la 
tecnología digital, ellos están permanentemente interconectados por medio del internet, el sitio web y los dispositivos móviles (Salamanca, 2011). El uso del internet, las innovaciones tecnológicas y las actuales tendencias sociales han modificado la visión y la forma de vivir de las personas, generando nuevas formas de compra, este hecho está impulsando a las organizaciones a innovar e implementar nuevas prácticas empresariales (Johnston \& Marshall, 2004); (SoftDoit, 2016); (Wachner, Plouffe, \& Grégoire, 2009).

Es de suma importancia tomar en cuenta el evolutivo e innovador avance tecnológico e implementar en forma adecuada dicha tecnología para el mejoramiento de la calidad del servicio electrónico en el sector hotelero de las PYMES de Cusco-Perú, es un hecho innegable que las micros, pequeñas y medianas empresas se hayan constituido en la base de la economía mundial; muchas de estas organizaciones con el paso del tiempo no han logrado desarrollarse o sobrevivir, enfrentando a grandes dificultades o teniendo un desarrollo muy lento, pero otras empresas sí han logrado crecer y tener éxito (Weber, 2010).

Estudios sobre el tema muestran una gran mortalidad en cuanto al desarrollo de las PYME, que en su gran mayoría fracasan y/o no superan los 5 años de existencia y un indicador, aún más sobrecogedor, es el hecho de que más del $90 \%$ de estas empresas no logran sobrevivir más allá de los 10 años, debido a muchos factores, entre ellos por la ausencia de una buena mercadotecnia digital y lo más preocupante por la impericia en la aplicación de dicha herramienta. Por otra parte, también se observa que el sector hotelero se ve obligado a aceptar las demandas costosas de las agencias de viaje online - OTAS-, teniendo como efecto en los hoteles, la disminución de sus precios en la oferta de sus servicios (Serrano, 2016).

Asimismo, las PYMES en general se constituyen en un factor esencial para la economía del Perú, por consiguiente en la investigación que se presenta, señala uno de los factores de la mercadotecnia digital que incide de manera significativa en la calidad de servicio electrónico del sector hotelero de las PYMES de Cusco-Perú, a fin de que estas organizaciones empresariales puedan utilizarlas para sobrevivir, crecer, desarrollarse y tener éxito en un escenario complejo y muy competitivo, permitiéndoles lograr una mayor solidez económica y financiera, fortaleciendo de esta manera a este sector; es por esa consideración que, el presente estudio investiga la incidencia de las redes sociales en la calidad del servicio electrónico en el sector hotelero de las PYMES de Cusco-Perú.

Cusco, es una ciudad emblemática no solo en el aspecto histórico y cultural, sino en su capacidad de aportar de manera significativa a la economía del país, siendo considerado como patrimonio cultural de la humanidad, es un centro muy importante de atracción turística, por lo que el sector hotelero está muy bien posicionado como actividad productiva fundamental de la región y el país. Machu Picchu recibe más de 1'100,000 de turistas anualmente. El 85\% del turismo receptivo nacional se dirige al circuito turístico del Sur, donde se encuentra la ciudad del Cusco y teniendo en cuenta un crecimiento promedio de $10 \%$ anual, se proyecta que para el año 2,021 la cantidad de visitantes será de 10 millones de turistas, este hecho generará una fuerte demanda de turismo receptivo, los mismos que requerirán los servicios de hospedaje de los hoteles, de igual forma estimulará un crecimiento económico significativo a nivel país y en vías de desarrollo como es el Perú, por consiguiente las PYMES de este sector desempeñan un rol fundamental en la dinámica del mercado, favoreciendo la generación de empleo y un efecto multiplicador importante en la economía regional y nacional (PROMPERU, 2014), (MINCETUR, 2014).

El objetivo general de la investigación es analizar la incidencia de las redes sociales en la calidad de servicio electrónico que ofrecen las PYMES del sector hotelero de Cusco, Perú; este estudio beneficiará a los gerentes, administradores y dueños de las pequeñas y medianas empresas de este sector, generando información para poder desarrollarse en una era digital competitiva, y en consecuencia mejorar la calidad de servicio electrónico. La hipótesis que se señala para esta investigación se centra en: las redes sociales incide en la Calidad de Servicio Electrónico en el sector hotelero de las PYMES de Cusco-Perú.

\section{Material y Métodos}

Se llevó a cabo un estudio de investigación cuantitativa, recolectando los datos a través de una encuesta para probar la hipótesis de investigación con base en la medición numérica y el análisis estadístico. En cuanto al tipo de investigación está determinado como exploratoria, descriptiva, correlacional y explicativa (Hérnandez, Fernández, \& Baptista, 2014). Esta investigación es no experimental en razón de que no hubo manipulación de datos, se limita a la observación de los hechos tal y como ocurren en la realidad. Las principales técnicas utilizadas para la recolección de datos son la documental, bibliográfica y de campo.

La población que se ha seleccionado fueron los principales hoteles PYMES en Cusco-Perú que corresponde a la categoría de tres (03) estrellas, los mismos que están registrados en la Dirección Regional de Comercio Exterior y Turismo —DIRCETUR— llegando a un total de 82 Hoteles formales. Ministerio de la Producción (2014). La encuesta estuvo dirigida a los huéspedes quienes son los que reciben el servicio brindado por los hoteles y califican la calidad del servicio electrónico recibido, del mismo modo también son los que adquieren y 
efectúan las reservas, teniendo contacto con la empresa en forma digital, por lo tanto, para establecer el número total de huéspedes a encuestar, se determinó el promedio de 12 huéspedes por día, esto a su vez multiplicado por el número total de hoteles formales registrados en DIRCETUR (82 hoteles), lo que da un resultado total de 984 huéspedes. Aplicando la fórmula del tamaño óptimo de la muestra, esta fue de 200 huéspedes de los hoteles de 3 estrellas de la ciudad de Cusco-Perú, con un promedio de entre 2 a 4 huéspedes encuestados por hotel (DIRCETUR, 2019).

En base al marco teórico se formuló la encuesta, habiéndose realizado una cuidadosa revisión bibliográfica de las variables analizadas; se integraron y adaptaron varios ítems de otras investigaciones que miden las variables propuestas. En el instrumento se utilizó una escala de Likert en donde 1 es nada de acuerdo, 2 poco de acuerdo, 3 ni de acuerdo ni en desacuerdo, 4 de acuerdo y 5 totalmente de acuerdo. Los datos obtenidos de la ficha de recolección de datos han sido transferidos al Microsoft Excel y luego al programa SPSS para su desarrollo estadístico.

\section{Resultados}

Se puede observar en la tabla 1 que existe correlación de un 52.6\% entre las redes sociales y la calidad de servicio electrónico de los hoteles de 3 estrellas de Cusco-Perú. El modelo es aceptable y significativo en un 27.6\%. Durbin-Watson nos muestra que las observaciones son independientes una de otra, no existe autocorrelación.

Tabla 1

Resultados de la regresión lineal

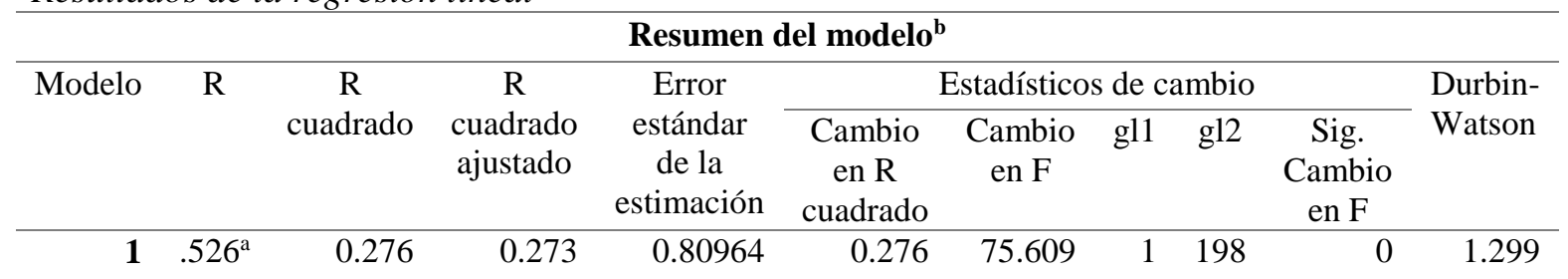

a. Predictores: (Constante), Redes Sociales

b. Variable dependiente: Calidad de Servicio Electrónico

Fuente: SPSS-V18.

De acuerdo a las significancias de la T-student, considerando un error estimado menor al 5\% (ver tabla 2), se concluye que existe un modelo aceptable, debido a que el constructo independiente Redes Sociales impacta significativamente al constructo dependiente Calidad de Servicio Electrónico, en un $46.2 \%$.

Tabla 2

Índice de factor de inflación de varianza

\begin{tabular}{|c|c|c|c|c|c|c|}
\hline \multicolumn{7}{|c|}{ Coeficientes $^{\mathbf{a}}$} \\
\hline & \multirow[t]{2}{*}{ Modelo } & \multicolumn{2}{|c|}{$\begin{array}{l}\text { Coeficientes no } \\
\text { estandarizados }\end{array}$} & \multirow{2}{*}{$\begin{array}{c}\text { Coeficientes } \\
\text { estandarizados } \\
\text { Beta }\end{array}$} & \multirow[t]{2}{*}{$\mathrm{T}$} & \multirow[t]{2}{*}{ Sig. } \\
\hline & & B & Desv. Error & & & \\
\hline \multirow[t]{2}{*}{1} & (Constante) & 1.492 & 0.168 & & 8.88 & .000 \\
\hline & $\begin{array}{l}\text { Redes } \\
\text { Sociales }\end{array}$ & 0.462 & 0.053 & 0.526 & 8.695 & .000 \\
\hline
\end{tabular}

a. Variable dependiente: Calidad de Servicio Electrónico

Fuente: SPSS-V18. 
La ecuación de regresión resultante del análisis entre las redes sociales como variable independiente y la calidad de servicio electrónico como variable dependiente (Y) es:

$$
\hat{\mathrm{Y}}=1.492+(0.462) \text { REDES SOCIALES }+\varepsilon
$$

\section{Discusión}

Se concuerda con la investigación que realizó Majó, Moya, \& Vall-Llosera (2018) sobre el impacto de las Redes Sociales en los ingresos de los Hoteles en Colombia, Ecuador y Perú, donde las redes sociales influyen en el pago y/o reservación de habitaciones; por consiguiente al tener una buena calidad de servicio electrónico mediante el uso eficiente de la mercadotecnia de las redes sociales se generarán mayores ingresos económicos para el sector hotelero, así como también buena comunicación con los usuarios que adquieran dicho servicio.

De igual forma también se tiene la investigación de Uribe Saavedra el cual menciona en su estudio titulado "Uso de las redes sociales digitales como herramienta de marketing", que los comentarios en las plataformas digitales inciden en la intención de compra y/o reserva de algún servicio, así como también se tiene la posibilidad de aportar e intercambiar información sobre productos, servicios y los intereses comunes que tienen los usuarios, dicha información es clave para ayudar a las empresas a mejorar su publicidad y promoción; por lo tanto, se concuerda con el autor, ya que al generar información esta también es valiosa para incrementar la calidad de servicio electrónico, en consecuencia se resalta la importancia de gestionar las redes sociales (Uribe Saavedra, 2014).

\section{Conclusiones}

Primera. En cuanto se obtuvieron los resultados se concluyó que las redes sociales inciden significativamente en la Calidad de Servicio Electrónico, ya que la significancia es inferior al 5\%, por lo que se acepta la hipótesis de investigación planteada anteriormente.

Segundo. A pesar de que Cusco-Perú es una Ciudad altamente turística, y hay aglomeración de turistas que visitan la ciudad imperial, en base a los resultados se observó que los hoteles hacen mediano uso de las redes sociales, lo cual es alarmante, ya que están dejando pasar muchas oportunidades de influir en la compra y/o reserva de habitaciones; por consiguiente, para incrementar la calidad de servicio electrónico mediante las redes sociales, este se podrá lograr impulsando el uso eficaz de esta herramienta tecnológica, que permita a la empresa realizar publicaciones de manera permanente, generando una presencia más significativa en estos medios digitales, y así poder tener mayor influencia en la reserva de habitaciones realizadas por el cliente, utilizando eficientemente los medios sociales como Facebook, Instagram, twitter, entre otros; así, a través de ellos se puedan promover, eficientemente, ofertas y campañas promocionales para que los huéspedes compren y/o reserven habitaciones y todo ello para beneficio de los hoteles y su calidad de servicio electrónico.

Tercera. Cabe resaltar la importancia de seguir estudiando las redes sociales como futura línea de investigación, y asi seguir aportando de manera práctica en la calidad del servicio electrónico, ya que estas seguirán avanzando y actualizando su uso, todo debido a los avances tecnológicos y sociales.

\section{Referencias bibliográficas}

DIRCETUR. (15 de Septiembre de 2019). Dirección Regional de Comercio Exterior $y$ Turismo . Obtenido de http://www.dirceturcusco.gob.pe/

Hérnandez, R., Fernández, C., \& Baptista, M. (2014). ¿Qué características posee el enfoque cuantitativo de investigación? En Metodología de la Investigación (pág. 4). Distrito Federal: McGraw-Hill / Interamericana Editores, S.A de C.V.

Johnston, M. W., \& Marshall, G. W. (2004). Administración de Ventas. México: McGRAW-HILL/Interamericana Editores, S.A. de C.V.

Majó, J., Moya, D., \& Vall-Llosera, L. (2018). Impacto de las Redes Sociales en los ingresos de los Hoteles en Colombia, Ecuador y Perú. Facultad de Ciencias Económicas: Investigación y Reflexión, vol. XXVI.

MINCETUR. (2014). Ministerio de Comercio Exterior y Turismo. Obtenido de www.mincetur.gob.pe

Producción, M. d. (2014). Ministerio de la Producción. Obtenido de http://www.produce.gob.pe/remype/data/mype2014.pdf
PROMPERU. (2014). Obtenido de ¿Qué es el turismo? https://www.lampadia.com/assets/uploads_documentos/e783bque-es-el-turismo-turismo.pdf

Salamanca, C. (20 de Septiembre de 2011). Marketing digital como canaldepromocion y venta. caso sitio Web escuela de posgrado de Marketing Internacional. La Plata, Buenos Aires, Argentina: Universidad Nacional de La Plata.

Serrano, L. d. (17 de noviembre de 2016). Mglobal. Obtenido de http://mglobalmarketing.es/blog/las-consecuencias-deprescindir-de-un-plan-de-marketing-para-las-pymes/

SoftDoit. (28 de Junio de 2016). Obtenido de II estudio sobre las Herramientas de Marketing \& Ventas y el uso del software CRM en las empresas españolas en 2016: https://www.softwaredoit.es/estudio/estudio-herramientasmarketing-ventas-uso-software-crm-empresas-espana-2016.html 
Uribe, Á. F. (Mayo de 2014). Uso de las redes sociales digitales como herramienta de marketing. Bellaterra, Barcelona: Universidad Autónoma de Barcelona .

Wachner, T., Plouffe, C. R., \& Grégoire, Y. (2009). Industrial Marketing Management. Obtenido de SOCO's impact on individual sales performance: The integration of selling skills as a missing link: http://chairemarketingservices.hec.ca/wp-

content/uploads/2015/05/WachnerPlouffegregoire.pdf
Weber, L. (2010). cómo las comunidades de consumidores digitales construyen su negocio. En Marketing en las redes sociales. México: Mc Graw-Hill Interamericana de editores S.A. de C.V. segunda edición. 